\title{
Feasibility of Transmit Diversity for IS-136 TDMA Systems
}

\author{
Ying-Chang Liang \\ Centre for Wireless Communications, National University of Singapore \\ 20 Science Park Road, Singapore 117674 Email: cwclyc@leonis.nus.edu.sg \\ Ye (Geoffrey) Li \\ AT\&T Labs - Research, 100 Schulz Dr., Rm. 4-152 \\ Red Bank, NJ 07701-7033, USA Email: liye@research.att.com \\ K. J. Ray Liu \\ Department of Electrical Engineering, University of Maryland \\ College Park, MD 20742, USA Email: kjrliu@isr.umd.edu
}

\begin{abstract}
In this paper, we investigate the performance of transmit diversity technique operating in IS-136 TDMA systems where the Doppler frequency can be as high as $184 \mathrm{~Hz}$. Transmit diversity transforms a flat fading channel into a frequency selective fading channel, thus we use finite length decision feedback equalizers to estimate the desired signal at the receiver. The channel parameters are trained via the training symbols and tracked using decision-directed symbols. It is shown that systems with transmit diversity can provide a significant performance improvement over systems without transmit diversity for Rayleigh flat fading channels when the Doppler frequency is less than $40 \mathrm{~Hz}$; however for systems with high Doppler frequency, transmit diversity alone cannot totally mitigate the fast fading effect since the effects of the channel estimation errors may cancel the benefit of the transmit diversity.
\end{abstract}

\section{INTRODUCTION}

In mobile wireless communication systems, the transmission quality is usually limited by multipath fading and fast movement of mobile users. Spatial diversity is a well-known technique to combat such fading effects. Two types of spatial diversity can be used: receive diversity and transmitdiversity [1]. In receive diversity systems, multiple antennas are equipped at the receiver to acquire multiple copies of the transmitted signal which experiences independent fading channels. Receive diversity is a powerful technique for mitigating rapid fading, suppressing intersymbol interference (ISI) and even cochannel interference (CCI), and can be easily implemented in mobile-to-base (up-

The work was done when he worked at University of Maryland at College Park. link) transmission in cellular systems. However, this technique is impractical in base-to-mobile (downlink) transmission due to implementation difficulty. In such settings, transmit diversity can be used to improve the downlink transmission quality. On the other hand, in the applications where receive diversity is applicable, transmit diversity can also be exploited in conjunction with receive diversity to provide further improved performance! e even when a few antennas at the base and mobile unit are used.

Many schemes have been proposed to implement transmit diversity technique for wireless communication systems. When the downlink channel fading parameters are known, we may obtain the best SNR at the receiver by choosing a proper set of transmit weights [2]. Although probing-feedback approach can be used to measure the channel parameters, this method is inefficient when the Doppler frequency is high, especially in IS-136 TDMA systems. In [3], multiple antennas transmit delayed versions of a signal, creating frequency-selective fading, which is equalized at the receiver to provide diversity gain. More general approach which extends the delayed operation to a class of linear transforms were proposed in $[4,5]$.

This paper investigates the performance of a transmit diversity system operating in a mobile environment with rapid fading channels. Our analysis is based upon the transmit diversity technique presented in [5]. We first present a general transmit diversity system model, then focus on the feasibility of transmit diversity for IS-136 TDMA systems in which the Doppler frequency may be as high as $184 \mathrm{~Hz}$ when 
the carrier frequency is $2 \mathrm{GHz}$ and the mobile user is moving at 60 miles/hour. Since the channels parameters are time-varying within one IS-136 time-slot, we use adaptive recursive least-square (RLS) algorithm to track the channel parameters. Monte Carlo simulations show that transmit diversity systems have better performance than systems without transmit diversity for Rayleigh flat fading channels, when the Doppler frequency is less than $40 \mathrm{~Hz}$. However, when the Doppler frequency is getting higher, the effects of the channel estimation errors may cancel the benefits of transmit diversity, thus systems with transmit diversity may work poorer than systems without diversity. Based on this fact, we suggest to use both transmit and receive diversity for IS-136 TDMA systems with rapid flat fading channels. Simulations show that systems with !2 transmit antennas and 3 receive antennas can attain $10^{-2}$ BER performance when the Doppler frequency is $184 \mathrm{~Hz}$ and signal-to-noise (SNR) is $20 \mathrm{~dB}$.

\section{Transmit Diversity for IS-136 TDMA SYSTEMS}

Suppose that $M$ antennas are equipped in the transmitter and the transmitted signal from the $m$ th antenna is denoted in baseband form by $y_{m}[n], m=$ $0,1, \cdots, M-1$. For convenience, we consider the flat fading channel case only, although the results can be extended to frequence-selective fading channel case immediately.

The received signal at the receiver is given by

$$
r[n]=\sum_{m=0}^{M-1} a_{m} y_{m}[n]+w[n]
$$

where $a_{m}$ stands for the flat fading coefficient of the $m$ th channel, and $w[n]$ denotes the receiver noise, which is complex-valued, zero-mean white Gaussian noise with variance $N_{0}$. We assume that the fading coefficients $a_{0}, a_{1}, \cdots, a_{M-1}$ are mutually independent, complex valued, zero-mean Gaussian random variables with variance $\sigma_{a}^{2}$. In each transmit antenna, the transmitted signal $y_{m}[n]$ is obtained by mapping the desired signal $x[n]$ through a linear transform,

$$
y_{m}[n]=\sum_{k=-\infty}^{\infty} h_{m}[k] x[n-k]
$$

where $h_{m}[n]$ is called the $m$ th antenna signature, whose associated Fourier transform is $H_{m}(\omega)=$ $\sum_{k=-\infty}^{\infty} h_{m}[k] e^{-\jmath \omega k}$. Hence, the received signal can be further expressed as

$$
r[n]=x[n] * g[n]+w[n]
$$

where

$$
g[n]=\sum_{m=0}^{M-1} a_{m} h_{m}[n]
$$

is the impulse response of the effective channel generated by the antenna precoding. This channel has frequency response

$$
G(\omega)=\sum_{m=0}^{M-1} a_{m} H_{m}(\omega)
$$

Note if there is only one transmit antenna, the channel is just a flat fading channel, thus linear transform operation effectively transforms the original nonselective fading channel into the frequency selective fading channel, which makes transmit diversity systems be able to mitigate multipath fading effects.

The antenna signatures should satisfy the conditions

$$
\sum_{m=0}^{M-1}\left|H_{m}(\omega)\right|^{2}=1
$$

and

$$
\sum_{m=0}^{M-1} H_{m}(\omega) H_{m}^{*}(\mu) \rightarrow 0
$$

when $\omega \neq \mu$ and $M \rightarrow \infty$. Based on these requirements, four admissible sets of signatures were proposed in [5]: ideal bandpass filter, one-symbol delay filter, exponential filter and Hardmard filter. Note for any admissible filter and any number of antennas, the summation of the transmitted powers are kept the same, which means that no additional powers are needed.

\section{Channel Parameter Tracking}

Since the received signal at the receiver is the output of a frequency-selective fading channel driven by the desired signal, when the fading coefficients are known, the "effective" channel impulse response can be computed, and linear equalizer (LE) or decision feedback equalizer (DFE) can be implemented to estimate the desired signal. Specifically, we focus on finite length DFE. Let the equalizer output be

$$
\widehat{x}[k]=\sum_{j=-K_{1}}^{0} c[j] r[k-j]+\sum_{j=1}^{K_{2}} c[j] \tilde{x}[k-j]
$$


where $\widehat{x}[k]$ is an estimate of the $k$ th information symbol, $c\left[-K_{1}\right], \cdots, c[0]$ are the feedforward coefficients, and $c[0], \cdots, c\left[K_{2}\right]$ are the feedback coefficients, and $\tilde{x}[k-1], \cdots, \tilde{x}\left[k-K_{2}\right]$ are previously detected symbols. Based on minimum MSE criterion, the DFE tap coefficients are related to the "effective" channel parameter $g[n]$ by

$$
\sum_{j=-K_{1}}^{0} \phi[l, j] c[j]=g^{*}[-l], \quad l=-K_{1}, \cdots,-1,0
$$

where

$$
\begin{aligned}
\phi[l, j]= & \sum_{m=0}^{-l} g^{*}[m] g[m+l-j]+N_{0} \delta[l, j], \\
& l, j=-K_{1}, \cdots,-1,0
\end{aligned}
$$

and

$$
c[k]=-\sum_{j=-K_{1}}^{0} c[j] g[k-j], \quad k=1,2, \cdots, K_{2}
$$

We consider two sets of antenna signatures: onesymbol delay filter and Hardmard filter. For these two kinds of filters, the "effective" channels are $(M-1)$ order FIR filters, thus from (11) only $(M-1)$ feedback coefficients are needed.

Since the channel parameters can change fast within one IS-136 time slot, the channel parameters must be estimated and tracked adaptively via training symbols and decision directed symbols.

Another simple method is to estimate the equalizer coefficients directly. Let $\mathbf{r}[n]$ denote the observation vector at time $n$, and $\mathbf{c}[n]$ denote the parameter vector at time $\mathrm{n}$. Note $\mathbf{r}[n]$ consists of the outputs at the receiver and the previous decided symbols for $k<n$. The parameter vector $\mathbf{c}[n]$ can be found by minimizing

$$
C(\mathbf{c}[n])=\frac{1}{Q} \sum_{k=1}^{N}\left|\mathbf{c}^{H}[n] \mathbf{r}[n-k]-x[n-k]\right|^{2}
$$

where $Q$ is the window length.

Direct calculation yields that the optimum parameter vector $\mathbf{c}[n]$ is given by

$$
\mathbf{c}[n]=\mathbf{R}^{-1}[n] \mathbf{p}[n]
$$

where

$$
\mathbf{R}[n]=\frac{1}{Q} \sum_{k=1}^{N} \mathbf{r}^{H}[n-k] \mathbf{r}[n-k]
$$

and

$$
\mathbf{p}[n]=\frac{1}{Q} \sum_{k=1}^{N} \mathbf{r}[n-k] \bar{x}^{*}[n-k]
$$

where $\bar{x}[m]=x[m]$ if $m$ is less than or equal to the length of training sequence, and $\bar{x}[m]=\tilde{x}[m]$ if $m$ is larger than the length. When the channel parameters change slowly, the longer the window length is, the more accurate the tracked parameters are. However, in order for the DFE to accurately track fast fading channel parameters, the window length cannot be too long. On the other hand, in IS-136 TDMA systems, the training sequence contains only 14 symbols, thus the window length $N$ is usually less than or equal to 14.

\section{Performance Evaluation Throvgh Simulation}

Transmit diversity is applied to mobile wireless communication environment. Especially, IS-136 TDMA systems with high Doppler frequency are considered. Adaptive RLS algorithm is exploited to equalize the received signal using 14 symbol training sequence followed by decided symbols. The 134 transmitted symbols are randomly drawn from $\left\{\frac{1+j}{\sqrt{2}}, \frac{1-j}{\sqrt{2}}, \frac{-1+j}{\sqrt{2}}, \frac{-1-j}{\sqrt{2}}\right\}$. DQPSK modulation is used with coherent detection. The parameters of the equalizer are initially estimated by using the training sequence, and after the training period, they are tracked via decision-directed symbols. Monte Carlo simulations are performed over 1000 time slots. For transmit diversity system, two transmit antennas are used and one-symbol delay filter signatures are implemented. The reason why we use two transmit antennas is that when the transmit antenna number increase from 1 to 2 , we obtain the largest performance improvement! while when the antenna number further increases, the performance improvement becomes lesses and lesser, and moreover, this may result in a difficulty in implementing the equalizer. At the receiver, a onefeedback-tap and three-feedforward-tap DFE is employed to estimate the desired signal.

Figure 1 shows the BER versus SNR for different Doppler frequency $f_{d}$ and transmit antenna number $M$. Figure 2 shows BER versus Doppler frequency when the SNR is fixed at $18 \mathrm{~dB}$ and $20 \mathrm{~dB}$. It is seen that transmit diversity systems have better performance than systems without transmit diversity for 


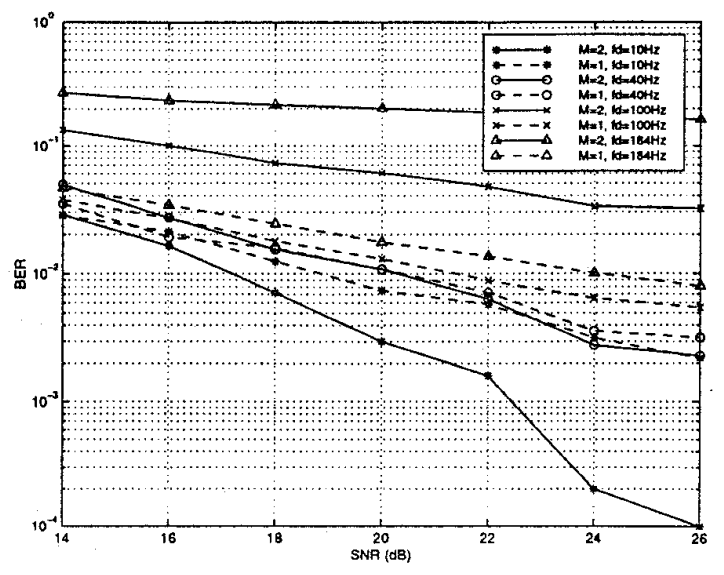

Fig. 1. BER versus SNR for different antenna number and Doppler frequency

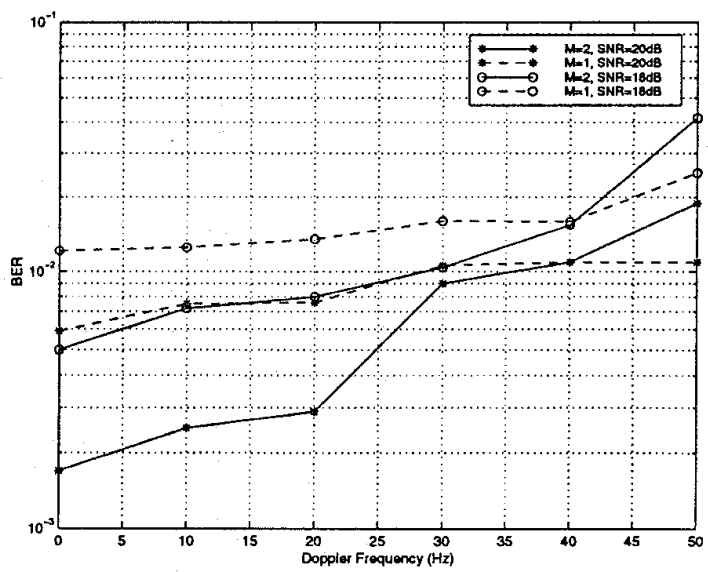

Fig. 2. BER versus Doppler frequency for different antenna number and SNR

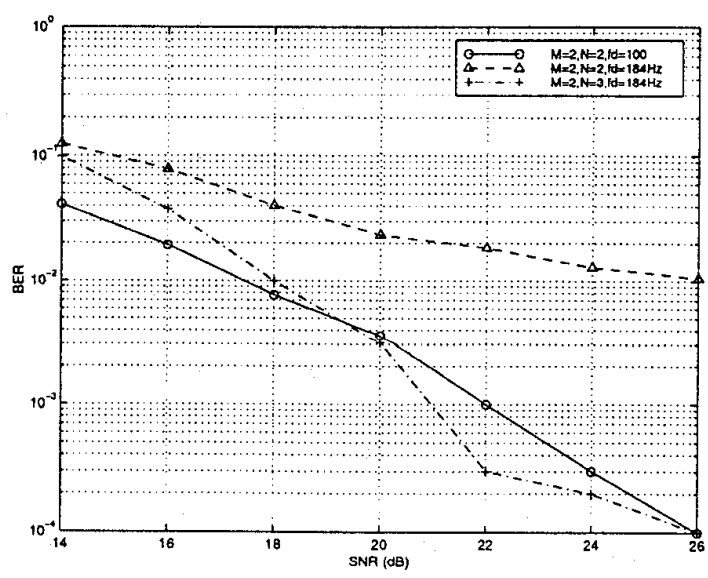

Fig. 3. BER versus SNR for different transmit and receive antenna numbers and Doppler frequency
Rayleigh flat fading channels, when the Doppler frequency is less than $40 \mathrm{~Hz}$. However, when the Doppler frequency increases, the performance improvement is not obvious, and on the other hand, systems with transmit diversity may work worse than systems without diversity for flat fading. This is because for high Doppler frequency, the transmit diversity system cannot track the channel parameters accurately, thus the DFE may not work well due to serious error propagation. Based on these observations, we suggest to use both transmit and receive diversity for IS-136 TDMA systems with rapid fading channels. Figure 3 shows BER verus SNR for different transmit antenna number $M$ and! receive antenna number $N$. It is seen that systems with 2 transmit antennas and 3 receive antennas can attain $10^{-2} \mathrm{BER}$ performance when the Doppler frequency is $184 \mathrm{~Hz}$ and SNR is $20 \mathrm{~dB}$.

\section{Conclusions}

In this paper we have investigated the performance of communication systems with transmit diversity. While transmitter diversity can provide a significant performance improvement over a system without diversity for Rayleigh flat fading channel when the Doppler frequency is less than $40 \mathrm{~Hz}$; for IS-136 systems with high Doppler frequency, transmitter diversity alone cannot totally mitigate the fast fading effect. This is because that the effects of the channel estimation errors may cancel the benefit of the transmit diversity. Hence, the combination of transmit diversity and receive diversity is a promising way in these systems.

\section{REFERENCES}

[1] J. H. Winters, J. Salz and R. D. Gitlin, "The impact of antenna diversity on the capacity of wireless communication systems," IEEE Trans. on Communications, vol.42, No.2/3/4, pp.1740-175, Feb./March/April 1994.

[2] D. Gerlach and A. Paulraj, "Adaptive transmitting antenna arrays with feedback," IEEE Signal Processing Letters, vol.1, pp.150-152, October 1994.

[3] A. Wittneben, "A new bandwidth efficient transmit antenna modulation diversity scheme for linear digital modulation," Proc. of IEEE ICC'93, pp.1630-1634, 1993.

[4] J. H. Winters, "The diversity gain of transmit diversity in wireless systems with Rayleigh fading," Proc. of IEEE ICC'94, pp.1221-1225, 1994.

[5] G. W. Wornell and M. D. Trott, "Efficient signal processing techniques for exploiting transmit antenna diversity on fading channels," IEEE Trans. Signal Processing, vol.45, pp.191-205, Jan. 1997. 